\title{
Side Effects of Immunosuppressive Regimens After Renal Transplantation and Multiple-Sample Assessment of Quality of Life
}

\author{
Siwen Wei, Na Yi, Lidao Bao* \\ College of Pharmacy, Inner Mongolia Medical University, Hohhot, P. R. China \\ Email address: \\ baolidao237@163.com (Lidao Bao) \\ ${ }^{*}$ Corresponding author
}

To cite this article:

Siwen Wei, Na Yi, Lidao Bao. Side Effects of Immunosuppressive Regimens After Renal Transplantation and Multiple-Sample Assessment of Quality of Life. American Journal of Clinical and Experimental Medicine. Vol. 5, No. 3, 2017, pp. 69-74.

doi: 10.11648/j.ajcem.20170503.13

Received: December 14, 2016; Accepted: April 5, 2017; Published: April 12, 2017

\begin{abstract}
Influences of the side effects and interferences of immunosuppressants on the quality of life (QOL) of renal transplant recipients have been discussed. 72 renal transplant recipients were surveyed utilizing 25 -item health status questionnaires, and modified transplant symptom occurrence and symptom distress scales. The results showed that the frequencies and symptom interferences of side effect after receiving immunosuppressants were below medium level. Physical and mental health related QOL was most significantly affected by symptom occurrence and symptom distress, respectively. Besides, postoperative comorbidity, work status, age and household income have also been demonstrated to be able to predict the postoperative QOL. Side effects all occurred to various extents after receiving immunosuppressants to the renal transplant recipients, the occurrence and interference of the symptoms were not apparent, which would affect the postoperative QOL.
\end{abstract}

Keywords: Renal Transplantation, Drug Side Effect, Rational Use, High Quality Nursing

\section{Introduction}

Since the last half century, renal transplantation has attracted worldwide attention in the medical field. According to incomplete statistics in China, approximately 150 thousand and 500 thousand patients die from uremia and type B nephritis associated end-stage renal diseases every year [1]. At present, success rate of kidney transplant surgeries in China is $85 \%$, 1-year and 3-year survival rates of the benign renal diseases after transplantations are $90 \%$ and $80.5 \%$, respectively $[2,3]$. Although the conditions of the renal transplant recipients have been fundamentally improved with basic daily self-care abilities and extended survival periods, QOL of the patients is still largely different from that of the normal people. The patients will confront physiological, psychological, social and many other problems and stresses. Notably, the patients need to take lifelong immunosuppressants to control the physical rejections, which will lead to series of side effects including influences on metabolism and organ function damages $[4,5]$. This study aimed to explore the impact of the side effects and their symptom distress brought about by postoperative immunosuppressive therapy after renal transplantations (abbreviated as immunosuppressive therapy below) on QOL.

\section{Objects and Methods}

\subsection{Research Objects}

Convenient sampling was utilized to select 100 cases of renal transplant recipients from several hospital renal transplant centers from January to December in 2010 with the criteria including: 1) postoperative survival time more than three months; 2) conscious enough to complete the surveys solely; 3) eligible to enroll in this research. Exclusion criterion: unconscious to complete the questionnaires. 100 questionnaires were distributed, 72 valid ones were returned, the returning rate was $72 \%$. Among the returned questionnaires, 42 patients were male $(58.33 \%)$ and 30 patients were female (41.67\%). All the patients aged from 12 
to 71 years old with the mean value of $41.26 \pm 11.72$. Religion: 70 patients did not have religions (97.22\%) and 2 patients had religions $(2.78 \%)$. Living arrangement: 6 patients lived alone $(8.33 \%), 62$ patients lived with their families $(86.11 \%), 4$ cases were missing (5.56\%). Per capita monthly household income: $<2000$ Chinese yuan in 24 cases (20.1\%), 2000-4000 Chinese yuan in 25 cases $(50.0 \%),>4000$ Chinese yuan in 20 cases $(26.0 \%), 2$ cases were missing (3.9\%). Payment method: self-expense in 15 cases $(20.83 \%)$, medical insurance in 45 cases $(62.50 \%)$, public expense in 6 cases $(8.33 \%), 6$ cases were missing (8.33\%). Postoperative time: 3-6 months in 12 cases $(16.67 \%), 7-12$ months in 4 cases $(5.56 \%), 1-3$ years in 45 cases $(32.5 \%), 4-6$ years in 5 cases $(6.94 \%), 7$ years and above in 6 cases $(8.33 \%)$. Postoperative comorbidity: did not occur in 56 cases $(77.78 \%)$, occurred in 13 cases $(18.06 \%), 3$ cases were missing $(4.17 \%)$. Postoperative work status: participated in social work in 22 cases $(30.56 \%)$, half worked and half rested in 21 cases (29.17\%), rested in 29 cases (40.28\%). Kidney source: relative kidney donors in 38 cases (52.78\%), cadaveric kidney donors in 34 cases $(47.22 \%)$.

\subsection{Research Tools}

\subsubsection{Self-Administered Questionnaires}

Demographics and disease-related information of the renal transplant recipients such as age, gender, ethnicity, religion, marital status, living arrangement, education, career, household income, payment method of medical expenses, with/without Type B nephritis, preoperative diagnosis, postoperative comorbidity, occurring time of postoperative acute rejection, regular rechecking status, postoperative work status and kidney donation source were included [6].

\subsubsection{Short form 36 Health Survey (SF-36)}

The 25-item scale used for the survey consisted of 5 parts including physical and mental health related QOL. The former was composed of 2 parts including physical function, role of physical function, body pain and general health, and the latter consisted of 5 terms including vitality, social function, role of emotional function and mental health. In these two parts, except that questions in the two parts concerning the roles of physical and emotional functions could be answered by "Yes" or "No", all the other questions included 4-5 levels. Each question was assigned a corresponding weight according to the damage severity of the representative function, and the resulting scores of various parts were transformed into the 100-mark system. Scores of each part ranged from 0 to 100 points, and higher scores referred to higher QOL. Reliability and validity of the scale were tested, and the consistency reliability coefficients of the scale all ranged from 0.52 to 0.78 except for the parts concerning social function and vitality. The reliability test was repeated two weeks later, yielding the values among the range of 0.46 to $0.84[7,8]$.

\subsubsection{Modified Transplant Symptom Occurrence and Symptom Distress Scale-59 Revised (MTSOSD-59R)}

The scale was designed by Dobbels et al. [9], which was used to measure a variety of side effects and symptom distresses of the immunosuppressive regimens after different transplantations [10] The scale consisted of 59 items in total, and each item was used to measure the symptom occurrence frequency of one certain side effect and that induced by the mental distress of renal transplant recipients. Measurements of the side effect frequencies were classified into five levels: 0 point for "not at all", 1 point for "occasionally", 2 point for "sometimes", 3 point for "frequently", 4 point for "always". Total score of each part was obtained by adding those of the individual items, and a larger score indicated more frequently occurred side effects. Measurements of side effect distresses were classified from "not at all" to "extremely" corresponding to the score range of $0-4$. Total score of each part was similarly obtained by adding those of the individual items, and a larger score referred to more distresses of side effects. If the occurring frequency of a specific side effect of a patient was reported as "not at all" ( 0 point), the corresponding symptom distress would not be included in the statistical analysis as the missing data $[11,12]$.

\subsection{Methods}

Questionnaires were dispensed by the investigators on the spot and guided uniformly, which were completed by the patients themselves and retrieved. The entire completion time was $30 \mathrm{~min}$.

\subsection{Statistical Analyses}

SPSS 14.0 statistical software was utilized to establish the database and perform statistical analyses. $T$ tests were employed to compare group means.

\section{Results}

\subsection{Univariate Analyses of the Postoperative QOL (Table 1)}

Table 1. Univariate analyses of the postoperative QOL (score, $x \pm s$ ).

\begin{tabular}{|c|c|c|c|}
\hline Item & & $\begin{array}{l}\text { Total score of } \\
\text { physiological health }\end{array}$ & $\begin{array}{l}\text { Total score of } \\
\text { mental health }\end{array}$ \\
\hline \multirow[t]{2}{*}{ Religion } & None & $287.52 \pm 65.27$ & $285.57 \pm 77.61 *$ \\
\hline & Yes & $305.13 \pm 59.91$ & $327.53 \pm 49.90$ \\
\hline \multirow{2}{*}{$\begin{array}{l}\text { Living } \\
\text { arrangement }\end{array}$} & Alone & $257.54 \pm 55.97 *$ & $251.78 \pm 71.89 *$ \\
\hline & With family & $294.11 \pm 64.72 *$ & $298.82 \pm 74.66$ \\
\hline \multirow[t]{2}{*}{$\begin{array}{l}\text { Postoperative } \\
\text { comorbidity }\end{array}$} & None & $303.60 \pm 57.77 *$ & $301.03 \pm 71.92 *$ \\
\hline & Yes & $255.26 \pm 70.85$ & $268.29 \pm 82.44$ \\
\hline \multirow[t]{3}{*}{$\begin{array}{l}\text { Household } \\
\text { income }\end{array}$} & $<2000$ & $274.38 \pm 63.66$ & $251.82 \pm 79.03 *$ \\
\hline & $2000-4000$ & $289.84 \pm 64.67$ & $299.32 \pm 73.18$ \\
\hline & $>4000$ & $300.65 \pm 67.24$ & $306.16 \pm 73.07$ \\
\hline \multirow[t]{5}{*}{$\begin{array}{l}\text { Postoperative } \\
\text { time/month }\end{array}$} & $3-6$ months & $230.62 \pm 30.37 *$ & $232.94 \pm 78.64$ \\
\hline & $7-12$ months & $274.06 \pm 58.98$ & $256.62 \pm 73.98$ \\
\hline & $13-36$ & $285.91 \pm 63.53$ & $299.38 \pm 67.70$ \\
\hline & $36-72$ & $295.15 \pm 69.41$ & $294.96 \pm 80.72$ \\
\hline & $>72$ & $316.30 \pm 38.47$ & $298.54 \pm 64.86$ \\
\hline \multirow[t]{3}{*}{$\begin{array}{l}\text { Postoperative } \\
\text { work status }\end{array}$} & $\begin{array}{l}\text { Participate in } \\
\text { social work }\end{array}$ & $313.79 \pm 54.51 *$ & $315.60 \pm 67.03 *$ \\
\hline & Half work & $283.04 \pm 68.48$ & $287.50 \pm 71.42$ \\
\hline & Rest & $277.38 \pm 65.32$ & $276.24 \pm 82.28$ \\
\hline
\end{tabular}

$* \mathrm{P}<0.05 ; * * \mathrm{P}<0.01$ 


\subsection{Side Effect Occurrences and Their Symptom Distresses of the Postoperative Immunosuppressants}

The overall occurring frequencies of 24 types of side effects of immunosuppressants were extremely low. The occurring frequencies and symptom distresses of the side effects scoring Top 10 are listed in Table 2.

Table 2. Top 10 side effect occurrences and symptom distresses of 72 cases of postoperative immunosuppressants (score, $x \pm s$ ).

\begin{tabular}{llllll}
\hline No. & Side effect & Score & No. & Symptom distress & Score \\
\hline 1 & Attention or memory problems & $1.27 \pm 1.53$ & 1 & Itchy skin & $1.29 \pm 1.43$ \\
2 & Increased hunger & $1.36 \pm 1.14$ & 2 & Decreased eye sight & $1.42 \pm 1.05$ \\
3 & Sleep disorder & $1.31 \pm 0.96$ & 3 & Sleep disorder & $1.24 \pm 0.96$ \\
4 & Thirsty & $1.27 \pm 1.04$ & 4 & Thirsty & $1.31 \pm 1.07$ \\
5 & Decreased eye sight & $1.27 \pm 1.01$ & 5 & Attention or memory problems & $1.28 \pm 1.88$ \\
6 & Itchy skin & $1.42 \pm 1.55$ & 6 & Abdominalgia & $1.17 \pm 1.11$ \\
7 & Dry skin & $1.16 \pm 1.23$ & 7 & Lack of vitality & $1.12 \pm 1.42$ \\
8 & Lack of vitality & $1.14 \pm 0.85$ & 8 & Diarrhea & $1.17 \pm 0.91$ \\
9 & Decreased interest in sex & $1.27 \pm 1.35$ & 9 & Abdominal distension & $1.14 \pm 1.07$ \\
10 & Fatigue & $1.06 \pm 0.97$ & 10 & Easy sweating & $1.24 \pm 0.87$ \\
\hline
\end{tabular}

\subsection{Correlations Between Symptom Occurrences and Symptom Distresses of the Postoperative Immunosuppressants and QOL (Table 3)}

Symptom occurrences and symptom distresses of the postoperative immunosuppressants were generally negatively correlated to each part of QOL, in which body pain was most significantly negatively correlated to both the symptom occurrences $(\mathrm{r}=-0.232, \mathrm{P}<0.001)$ and symptom distresses $(\mathrm{r}$ $=-0.624, \mathrm{P}<0.001)$.

Table 3. Correlations between symptom occurrences and symptom distresses of the postoperative immunosuppressants and QOL ( $r$ value).

\begin{tabular}{|c|c|c|c|c|c|c|c|c|c|c|c|c|}
\hline Item & SO & SD & PF & $\mathbf{R P}$ & BP & GH & VT & SF & RE & MH & TSH & ТРН \\
\hline SO & 1.000 & - & - & - & - & - & - & - & - & - & - & - \\
\hline SD & 0.637 & 1.000 & - & - & - & - & - & - & - & - & - & - \\
\hline PF & -0.275 & -0.320 & 1.000 & - & - & - & - & - & - & - & - & - \\
\hline RP & -0.264 & -0.241 & 0.275 & 1.000 & - & - & - & - & - & - & - & - \\
\hline BP & -0.542 & -0.576 & 0.395 & 0.326 & 1.000 & - & - & - & - & - & - & - \\
\hline $\mathrm{GH}$ & -0.377 & -0.260 & 0.268 & 0.240 & 0.325 & 1.000 & - & - & - & - & - & - \\
\hline SF & -0.467 & -0.421 & 0.321 & 0.394 & 0.496 & 0.444 & 0.528 & 1.000 & - & - & - & - \\
\hline $\mathrm{RE}$ & -0.281 & -0.342 & 0.278 & 0.571 & 0.371 & 0.191 & 0.483 & 0.354 & 1.000 & - & - & - \\
\hline $\mathrm{MH}$ & -0.362 & -0.282 & 0.376 & 0.279 & 0.347 & 0.465 & 0.654 & 0.541 & 0.452 & 1.000 & - & - \\
\hline TSH & -0.484 & -0.456 & 0.547 & 0.829 & 0.699 & 0.601 & 0.625 & 0.571 & 0.540 & 0.234 & 1.000 & - \\
\hline $\mathrm{TPH}$ & -0.482 & -0.459 & 0.547 & 0.550 & 0.533 & 0.472 & 0.818 & 0.748 & 0.781 & 0.232 & 0.154 & 1.000 \\
\hline
\end{tabular}

SO: symptom occurrence; SD: symptom distress; PF: physical function; RP: role of physical function; BP: body pain; GH: general health; VT: vitality; SF: social function; RE: role of emotional function; MH: mental health; TSH: total score of physical health; TPH: total score of psychological health

\subsection{Hierarchical Regression Analysis of the Postoperative QOL (Table 4)}

Hierarchical regression analysis was utilized in this study, and QOL related to physical health and mental health was used as the dependent variables. Statistically significant factors of general demographics and diseases, including religion (None $=0$, Yes $=1$ ), living arrangement $($ Alone $=0$, With family = 1), per capita monthly household income (dummy variable assignment method: reference variable was the value less than 2000 Chinese yuan), postoperative time (rating assignment method: $3-6$ months $=1,7-12$ months $=2$, $1-3$ years $=3,4-6$ years $=4,7$ years and above $=5$ ), postoperative work status (dummy variable assignment method: reference variable was the participation in social work) and age, were used as the first hierarchical level independent variables. Then symptom occurrence and symptom distress were considered in the second hierarchical layer, which was used to solely clarify their effects on the postoperative QOL. QOL related to postoperative physical health was affected by the factors including postoperative comorbidity, postoperative work status and symptom occurrence of side effect, which cooperatively accounted for $25.5 \%$ of the corresponding total variance. Meanwhile, QOL related to mental health was influenced by the factors including postoperative work status, age, monthly household income and symptom distress, which synergetically comprised $33 \%$ of the corresponding total variance. 
Table 4. Hierarchical regression analysis of the postoperative $Q O L$.

\begin{tabular}{|c|c|c|c|c|c|}
\hline \multirow[b]{2}{*}{ Dependent variable } & \multirow[b]{2}{*}{ Independent variable } & \multicolumn{2}{|l|}{ Model 1} & \multicolumn{2}{|l|}{ Model 2} \\
\hline & & $\begin{array}{l}\text { Regression } \\
\text { coefficient }\end{array}$ & $\begin{array}{l}\text { Standard regression } \\
\text { coefficient }\end{array}$ & $\begin{array}{l}\text { Regression } \\
\text { coefficient }\end{array}$ & $\begin{array}{l}\text { Standard regression } \\
\text { coefficient }\end{array}$ \\
\hline \multirow[t]{9}{*}{ Total score of physical health } & Constant & $11.361^{*}$ & - & $7.968^{*}$ & - \\
\hline & Living arrangement & 1.417 & 0.118 & 0.607 & 0.055 \\
\hline & Postoperative comorbidity & $-2.105^{* *}$ & -0.258 & $-1.438^{* *}$ & -0.178 \\
\hline & Half work half rest & $-1.709^{* *}$ & -0.216 & -1.241 & -0.172 \\
\hline & Rest & $-1.904^{* *}$ & -0.274 & $-1.625^{* *}$ & -0.256 \\
\hline & Age & 0.008 & 0.164 & 0.044 & 0.166 \\
\hline & Postoperative time & 0.000 & 0.007 & 0.001 & 0.013 \\
\hline & Symptom distress & - & - & -0.021 & -0.171 \\
\hline & Symptom occurrence & - & - & -0.102 & -0.157 \\
\hline \multirow[t]{11}{*}{ Total score of mental health } & Constant & $169.945^{*}$ & - & $237.287^{*}$ & - \\
\hline & Living arrangement & 32.114 & 0.157 & 17.461 & 0.123 \\
\hline & Postoperative comorbidity & -21.901 & -0.121 & -7.452 & -0.051 \\
\hline & Half work half rest & $-33.182^{* *}$ & -0.183 & $-24.324^{* *}$ & -0.158 \\
\hline & Rest & $-47.541^{* *}$ & -0.313 & -41.336 & -0.274 \\
\hline & Age & $2.014^{* *}$ & 0.640 & $1.670^{* *}$ & 0.301 \\
\hline & Religion & 23.524 & 0.148 & 22.514 & 0.145 \\
\hline & $2000-4000$ & $36.171^{* *}$ & 0.254 & $36.305^{* *}$ & 0.258 \\
\hline & $>4000$ & 34.305 & 0.183 & 26.92 & 0.163 \\
\hline & Symptom occurrence & - & - & -0.597 & -0.192 \\
\hline & Symptom distress & - & - & $-0.6543^{* *}$ & -0.252 \\
\hline
\end{tabular}

*) $\mathrm{P}<0.01$;**) $\mathrm{P}<0.05$; 3 ) Household income ${ }^{*}$

\section{Discussion}

\subsection{Relationships Between the Symptom Occurrence and Symptom Distress of Immunosuppressants and QOL of Renal Transplant Recipients}

Currently, investigations on the symptom occurrence and symptom distress of immunosuppressants were usually limited to lung and heart transplantations. In contrast, research on the renal transplantation is still seldom, which also suffers from small sample size, relatively early time, different research methods and unclear result expressions. Therefore, the latest modified version of the professional scale was used herein to measure the symptom occurrence and symptom distress of immunosuppressants, which was of large sample size compared to the previous research on the renal transplant patients [13-15]. Similar results were obtained compared to those of the previous studies, including a variety of side effects after taking immunosuppressants and occurring frequencies below the medium level. Among the side effects, the occurrences of crisp skin, increased hunger, fatigue, body change, mood disturbance, face change, decreased interest in sex, headache and increased hair growth were more frequent, and they were of relatively severe symptom distresses [16, 17]. Thus, certain side effects would occur due to the instinct characteristics of the immunosuppressants. However, both the symptom occurrence and symptom distress were not very serious and basically lay in the tolerable range of human body. From the above aspects, the results of the study were slightly better than those of previous studies, which may be related to different surveyed gender ratio, immunosuppressive regimen methods and cultures [18]. Moreover, although immunosuppressants have been greatly improved in the enhancing performance and reducing side effects, the resulting physical and (or) mental uncomfortable symptoms of the recipients would be inevitable. QOL of the patients was mainly affected by their subjective experiences including discomfort or pathological change, which has been verified previously $[19,20]$.

The research herein will assist the clinical caregivers in the following three aspects: 1) Help the caregivers be aware of the effects of the symptom occurrence or symptom distress on QOL of patients, improve the attention concerning the side effects and their symptom occurrences, and enhance the pertinence and purposiveness [21]; 2) Guide the patients and their families to understand the side effects of immunosuppressants, increase awareness and instant responses to the side effects; 3 ) Select advantageous regimens for the patients, and provide evidence for the development of novel immunosuppressants as well.

\subsection{Relationships Between the Demographics and Other Disease Factors and QOL of Renal Transplant Recipients}

The results of hierarchical regression analysis indicated that QOL of the renal transplant recipients would be largely affected owing to the physical or psychologically traumas originated from the a variety of postoperative comorbidities. Thus, nurses should pay more attention to the observing and monitoring of postoperative comorbidities, and decrease the impact of them on QOL by means of early prevention, detection and treatment. However, the relationship between age and QOL found herein was not the same as that of the previous studies [22-24]. This study showed that age was correlated to the mental health related QOL positively, which may be attributed to more competitive pressure from the social environment that young people are confronting. Their 
expected normal life is greatly different from the reality, which will result in more psychological pressure that leads to poorer mental health related QOL [25]. As a result, nurses ought to pay more attention to the psychological issues of young patients, provide necessary psychological intervention and support, assist to improve the responding methods, and help them to improve QOL. In addition, returning to social work will facilitate the regain of self-esteem for the patients to enrich themselves, reinforce their self-perception, realize their social values again, enhance their postoperative confidence in life remarkably, and eventually improve their perception of QOL [26].

\section{Conclusion}

Furthermore, medical expenses also placed heavy burdens on both society and families. Treatments for patients are often limited by economic conditions. Wealthy patients are able to afford the huge medical expenses without considering the economic impact on their families. Contrarily, the patients in poor economic conditions not only suffer from the pains of diseases, but also take the family burdens into consideration that will result in depression, excessive mental pressure, relatively low QOL perceived. Therefore, basic expenses of immunosuppressive regimens should be ensured with increased medical insurances in order to relieve their mental stress and improve the operative QOL [26].

In summary, QOL changes of the renal transplant recipients are complicated including various operative factors. Considering that the sample size of this study is still not sufficiently large and the research time span is large, further investigations are in need to validate the acquired results.

\section{References}

[1] Guo FF, Shao ZQ, Yang WY, Wang GJ, Tan SF, He XF, et al. Clinical analysis of living related renal transplantation with donors older than 50 years in China. Transplant Proc 2010,42:2471-2476.

[2] Li QS, Huo WQ, Nie ZL, Wang HF, Liang PH, Jin FS. Acute femoral neuropathy following renal transplantation: a retrospective, multicenter study in China. Transplant Proc 2010,42:1699-1703.

[3] Li L, Sun Q. Renal transplantation in China: ten years of experience at Nanjing Jinling Hospital. Clin Transpl 2006:71-77.

[4] Winsett RP, Hathaway DK. Predictors of QoL in renal transplant recipients: bridging the gap between research and clinical practice. Posttransplant Quality of Life Intervention Study Group. ANNA J 1999,26:235-240.

[5] Mingardi G. Quality of life and end stage renal disease therapeutic programs. DIA-QOL Group. Dialysis quality of life. Int J Artif Organs 1998, 21:741-747.

[6] Padrao MB, Sens YA. Quality of life of living kidney donors in Brazil: an evaluation by the short form-36 and the WHOQOL-bref questionnaires. Clin Transplant 2009,23:621-627.
[7] Nowicki M, Zwiech R, Dryja P, Sobanski W. Autonomic neuropathy in hemodialysis patients: questionnaires versus clinical tests. Clin Exp Nephrol 2009,13:152-155.

[8] Ricci Z, Picardo S, Ronco C. Results from international questionnaires. Contrib Nephrol 2007, 156:297-303.

[9] Dobbels F, Moons P, Abraham I, Larsen CP, Dupont L, De Geest S. Measuring symptom experience of side-effects of immunosuppressive drugs: the Modified Transplant Symptom Occurrence and Distress Scale. Transpl Int 2008, 21:764-773.

[10] Cleemput I, Kesteloot K, Moons P, Vanrenterghem Y, Van Hooff JP, Squifflet JP, et al. The construct and concurrent validity of the EQ-5D in a renal transplant population. Value Health 2004, 7:499-509.

[11] Dobbels F, Decorte A, Roskams A, Van Damme-Lombaerts R. Health-related quality of life, treatment adherence, symptom experience and depression in adolescent renal transplant patients. Pediatr Transplant 2010, 14:216-223.

[12] Moons P, De Geest S, Abraham I, Cleemput JV, Van Vanhaecke J. Symptom experience associated with maintenance immunosuppression after heart transplantation: patients' appraisal of side effects. Heart Lung 1998, 27:315-325.

[13] Sun YJ, Li AP, Pan ZQ, Luo F, Zou LH. [Systematic review of penetrating keratoplasty rejection treated by immunosuppressants]. Zhonghua Yan Ke Za Zhi 2010, 46:1122-1127.

[14] Lee EK, Cham TM, Tseng PL. A retrospective study on the utilization of and expenditure for immunosuppressants for organ transplant recipients in Taiwan--updated to 2006. Transplant Proc 2010,42:961-965.

[15] Quteineh L, Verstuyft C. Pharmacogenetics in immunosuppressants: impact on dose requirement of calcineurin inhibitors in renal and liver pediatric transplant recipients. Curr Opin Organ Transplant 2010, 15:601-607.

[16] Chang JW, Tsai HL, Yang LY. Successful treatment of refractory septic arthritis caused by salmonella and staphylococcus aureus with preservation of graft function in a long-term renal transplant recipient by total withdrawal of immunosuppressants. Clin Nephrol 2010,73:72-75.

[17] Greco M, Vitagliano T, Fiorillo MA, Greto Ciriaco A. A New Technique of Upper Eyelid Blepharoplasty Using the Orbicularis Muscle Flap. Aesthetic Plast Surg 2011.

[18] Margolis DJ, Hoffstad O, Bilker W. Lack of association between exposure to topical calcineurin inhibitors and skin cancer in adults. Dermatology 2007, 214:289-295.

[19] Schonder KS, Mazariegos GV, Weber RJ. Adverse effects of immunosuppression in pediatric solid organ transplantation. Paediatr Drugs 2010, 12:35-49.

[20] Zhang Y, Wang Y, Zhang P, Zhang XD, Yang Y. Extended-release doxazosin for treatment of renal transplant recipients with benign prostatic hyperplasia. Transplant Proc 2009, 41:3747-3751.

[21] Leon-Salas B, Olazaran J, Muniz R, Gonzalez-Salvador MT, Martinez-Martin P. Caregivers' estimation of patients' quality of life (QoL) in Alzheimer's disease (AD): an approach using the ADRQL. Arch Gerontol Geriatr 2011, 53:13-18. 
[22] Okushi T, Yoshikawa M, Otori N, Matsuwaki Y, Asaka D, Nakayama T, et al. Evaluation of symptoms and QOL with calcium alginate versus chitin-coated gauze for middle meatus packing after endoscopic sinus surgery. Auris Nasus Larynx 2012, 39:31-37.

[23] Brocker KA, Alt CD, Corteville C, Hallscheidt P, Lenz F, Sohn C. Short-range clinical, dynamic magnetic resonance imaging and P-QOL questionnaire results after mesh repair in female pelvic organ prolapse. Eur J Obstet Gynecol Reprod Biol 2011, 157:107-112.

[24] Claerhout F, Moons P, Ghesquiere S, Verguts J, De Ridder D, Deprest J. Validity, reliability and responsiveness of a Dutch version of the prolapse quality-of-life (P-QoL) questionnaire. Int Urogynecol J 2010, 21:569-578.
[25] Mor V. QOL measurement scales for cancer patients: differentiating effects of age from effects of illness. Oncology (Williston Park) 1992, 6:146-152.

[26] Boyer L, Simeoni MC, Loundou A, D'Amato T, Reine G, Lancon C, et al. The development of the S-QoL 18: a shortened quality of life questionnaire for patients with schizophrenia. Schizophr Res 2010, 121:241-250.

[27] Okubo Y, Natsume S, Usui K, Amaya M, Tsuboi R. Low-dose, short-term ciclosporin (Neoral(R)) therapy is effective in improving patients' quality of life as assessed by Skindex-16 and GHQ-28 in mild to severe psoriasis patients. $J$ Dermatol 2011, 38:465-472. 